\title{
Construction and Chemical Profile on "Activity Fingerprint" of Citri Reticulatae Pericarpium from Different Cultivars Based on HPLC-UV, LC/MS-IT-TOF, and Principal Component Analysis
}

\author{
Guodong Zheng $\mathbb{D}^{1},{ }^{1}$ Yingxin Chao, ${ }^{1}$ Meixia Luo, ${ }^{1,2}$ Bin Xie, ${ }^{3}$ Dedong Zhang, ${ }^{3}$ Pingjun Hu, ${ }^{1}$ \\ Xiujuan Yang, ${ }^{1}$ Depo Yang $\left(\mathbb{1},{ }^{4}\right.$ and Minyan Wei ${ }^{1}{ }^{1}$ \\ ${ }^{1}$ Key Laboratory of Molecular Target \& Clinical Pharmacology and the State Key Laboratory of Respiratory Disease, \\ School of Pharmaceutical Sciences \& The Fifth Affiliated Hospital, Guangzhou Medical University, Guangzhou, China \\ ${ }^{2}$ The Affiliated Brain Hospital of Guangzhou Medical University, Guangzhou Huiai Hospital, Guangzhou, China \\ ${ }^{3}$ Tianda Pharmaceutical (Zhuhai) Co., Ltd., Zhuhai, China \\ ${ }^{4}$ School of Pharmaceutical Sciences, Sun Yat-sen University, Guangzhou, China
}

Correspondence should be addressed to Depo Yang; lssydp@mail.sysu.edu.cn and Minyan Wei; weiminyan@163.com

Received 2 October 2019; Accepted 8 January 2020; Published 3 March 2020

Academic Editor: Francesca Mancianti

Copyright ( $\odot 2020$ Guodong Zheng et al. This is an open access article distributed under the Creative Commons Attribution License, which permits unrestricted use, distribution, and reproduction in any medium, provided the original work is properly cited.

\begin{abstract}
Citri Reticulatae Pericarpium (CRP), known as Chenpi (CP) in Chinese, is a medicinal food for health and fitness. In order to find out the characteristic activity chemicals distinguishing various cultivars of CRP and provide a reference for effective development of citrus resources, an "activity fingerprint" of CRP from 21 different cultivars was established based on the evaluation of antitussive and expectorant activities. There were 18 common peaks in the HPLC fingerprint, of which 3 flavonoid glycosides and 14 polymethoxyflavonoids (PMFs) were identified by LC/MS-IT-TOF. Furthermore, five characteristic chemicals were determined and similarity calculation with principal component analysis (PCA) was combined together to compare the similarity and difference among various cultivars. The results showed that some other cultivars were also similar to C. reticulata "Chachi" except for C. reticulata "Tangerina" and C. reticulata "Dahongpao" recorded in Chinese Pharmacopoeia. Most importantly, the peels of C. reticulata "Shiyueju," C. reticulata "Ponkan," C. reticulata "Tribute," and C. reticulata "Bayueju," traditionally rarely used for medicinal food, were highly similar to that of C. reticulata "Chachi" and rich in bioactive flavonoids, which can be considered the effective medicinal resources of CRP.
\end{abstract}

\section{Introduction}

Citri Reticulatae Pericarpium (CRP), also known as Chenpi (CP) in Chinese, the dry mature peel of Citrus reticulata Blanco or its cultivated varieties [1], has been traditionally used as both nutritional food and herbal medicine for clinic treatment of cough with phlegm, vomiting and diarrhea, and stomach ache [2]. There are four cultivars of CRP officially listed in Chinese Pharmacopoeia (2015 version), such as $C$. reticulata "Chachi," C. reticulata "Dahongpao," C. reticulata "Tangerina," and C. reticulata "Unshiu." Among them, the desiccative ripe peel of Citrus reticulata "Chachi," called
Guang Chenpi (GCP) in Chinese and produced chiefly in Xinhui (Guangdong, China), is well known as a traditional genuine herb with high quality. However, there are no more uniform quality standards on CRP application and other nonlisted cultivars were often sold at best quality prices, resulting in a confusing market with uneven quality of CRP. Thus, it is necessary to establish a scientific method and find the characteristic activity chemicals distinguishing various cultivars of CRP for quality control.

High-performance liquid chromatography (HPLC) fingerprint analysis has recently been applied as a quality evaluation method with high specificity, stability, 
reproducibility, and integrity because a large number of samples could be well analyzed by describing the similarities and differences through similarity results $[3,4]$. Although it is a good assumption to evaluate and compare a great number of CRP samples through the HPLC fingerprint, previous studies mainly focused on a single cultivar of $C$. reticulata "Chachi" from different places of Guangdong Province or four recorded cultivars [5], while other nonrecorded but common cultivars have not been taken into consideration. It is more reliable to find out characteristics distinguishing varieties to protect the health of the CRP market when adopting as many different cultivars as possible for quality comparison. In addition, the HPLC fingerprint is just a kind of qualitative analysis and the compounds corresponding to its characteristic peak have not been profiled [6], so it is of significance to further study the characteristic components in combination with other identification and quantification techniques.

Flavonoids have been proved to be the main chemical components in CRP, extracted mostly from methanol, and distributed into two types: flavonoid glycosides and polymethoxyflavonoids (PMFs). And recently, pharmacological studies reported for individual flavonoids described antiinflammatory $[7,8]$, antitumor $[9,10]$, antibacterial $[11,12]$, and antioxidant activities $[13,14]$ and cardiovascular protection $[15,16]$. However, there are few reports about the pharmacological activity of the mixed CRP extract so that the HPLC fingerprint has not yet been proved to relate to active compounds. Moreover, it is uncertain whether the methanol extract of CRP (MECRP) has antitussive and expectorant activities. The antitussive and expectorant activity evaluation of the MECRP played an indispensable part of "activity fingerprint" for systematical quality control.

In this study, 88 samples including 21 different cultivars were used for quality analysis. The MECRP was firstly prepared on the animal models for antitussive and expectorant activity evaluation. HPLC and HPLC combined with ion-trap and time-of-flight mass spectrometry (LC/MS-ITTOF) were then applied to clarify the characteristic chemicals corresponding to each common peak in the fingerprint and determine the content of five main flavonoids. Finally, similarity calculation and principal component analysis (PCA) were combined to compare the similarity and difference among various cultivars. Overall, this effective method of "activity fingerprint" would not only contribute to establishing an excellent quality evaluation system of CRP to find out the characteristic activity chemicals distinguishing various cultivars but also provide a reference for the effective development of citrus resources.

\section{Materials and Methods}

2.1. Plant and Animal Materials. Eighty-eight batches of samples including 21 different cultivars were gathered from different major citrus-producing regions in China (Table 1). The sample S1 (Lot no. 20141016) is chosen as reference crude herb for the activity assay and method validation with the qualification of China's protected geographical indication product, provided by the natural planting base in
Luokeng Town by Xinbaotang Company. The voucher specimens, appraised by Prof. Bo Wu, have been deposited at the Laboratory of Pharmacognosy, Guangzhou Medical University, Guangdong Province, China.

SPF Kunming mice weighing 18 to $22 \mathrm{~g}$, half male and female, were provided by the Medical Experimental Animal Center of Guangdong Province (License SCXK[Yue] 20130002). Animals were fasted for 12 hours before the first and the final administration but given water ad libitum. All the experimental procedures were performed in accordance with the Regulation on the Administration of Laboratory Animals issued by the State Council of China. All animals were killed by cervical dislocation, and all efforts were made to minimize suffering.

2.2. Chemicals and Reagents. The positive control drug, dextromethorphan hydrobromide $(\mathrm{DH})$ tablets, for the antitussive activity assay was purchased from Baiyunshan Guanghua (China). The ambroxol hydrochloride (AH) tablets, used for the expectorant activity assay, were obtained from Boehringer Ingelheim (Shanghai, China). The solvents for HPLC and LC/MS-IT-TOF analysis, HPLC-grade acetonitrile and purified water, used after filtration through membranes, were procured from Honeywell (America) and Yibao (China), respectively. Hesperidin (Chengdu Must Bio-Technology, Chengdu, China), nobiletin (Chengdu Must Bio-Technology, Chengdu, China), 3,5,6,7,8,3', $4^{\prime}$ heptamethoxyflavone (Weikeqi, Sichuan, China), tangeretin (Chengdu Must Bio-Technology, Chengdu, China), and 5hydroxy-6,7,8,3', $4^{\prime}$-pentamethoxyflavone (Spring \& Autumn, Nanjing, China) were all of reference standard grade, with the high purity above $98 \%$. All other reagents were of analytical grade and pure.

2.3. Standard Solution and MECRP Preparation. Each reference of the five flavonoids was dissolved together in methanol at an appropriate concentration to obtain the mixed standard solution. Each sample powder was extracted ultrasonically with 100 times of methanol (w/v) for 30 minutes at $320 \mathrm{~W}$ [17]. The solvent of the sample S1 was eliminated by a rotary evaporator to obtain the dried MECRP (extraction rate $=31 \%, g / g$ ), which was dispersed to different concentrations with distilled water for the activity assay. Meanwhile, all the sample solutions with the MECRP were filtered through a $0.22 \mu \mathrm{m}$ membrane for HPLC-UV and LC/MS-IT-TOF analysis.

\subsection{Evaluation of Antitussive and Expectorant Activities of MECRP}

2.4.1. Antitussive Activity Assay. Fifty Kunming mice were randomly allocated to five groups of half male and half female each: control, MECRP-20, MECRP-40, MECRP-80, and $\mathrm{DH}-60$. The control group and standard group received normal saline $(0.9 \%)$ and $\mathrm{DH}(60 \mathrm{mg} / \mathrm{kg})$ daily for 7 continuous days, respectively, while the test groups were administered orally with the MECRP $(20,40$, and $80 \mathrm{mg} / \mathrm{kg})$. 


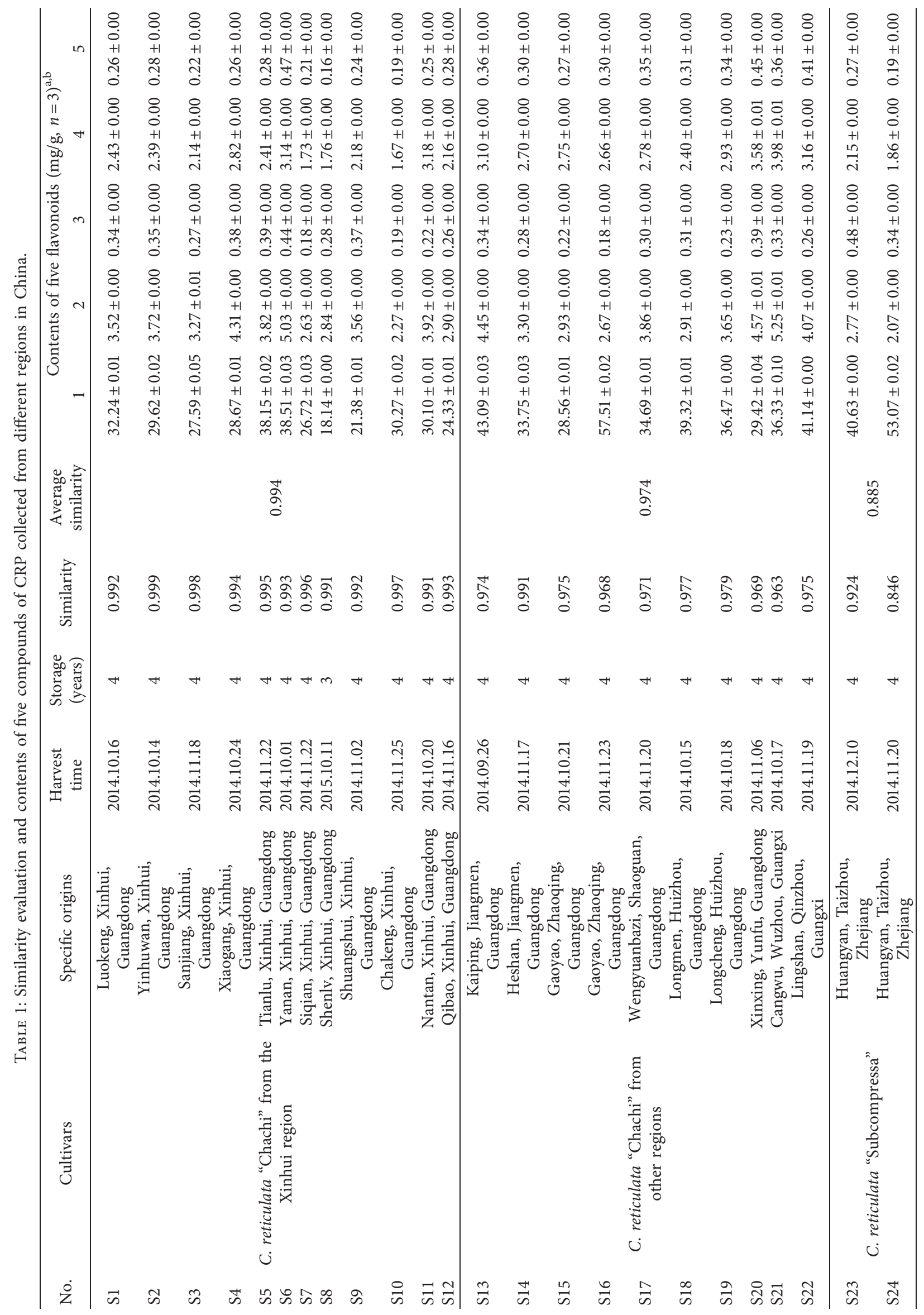




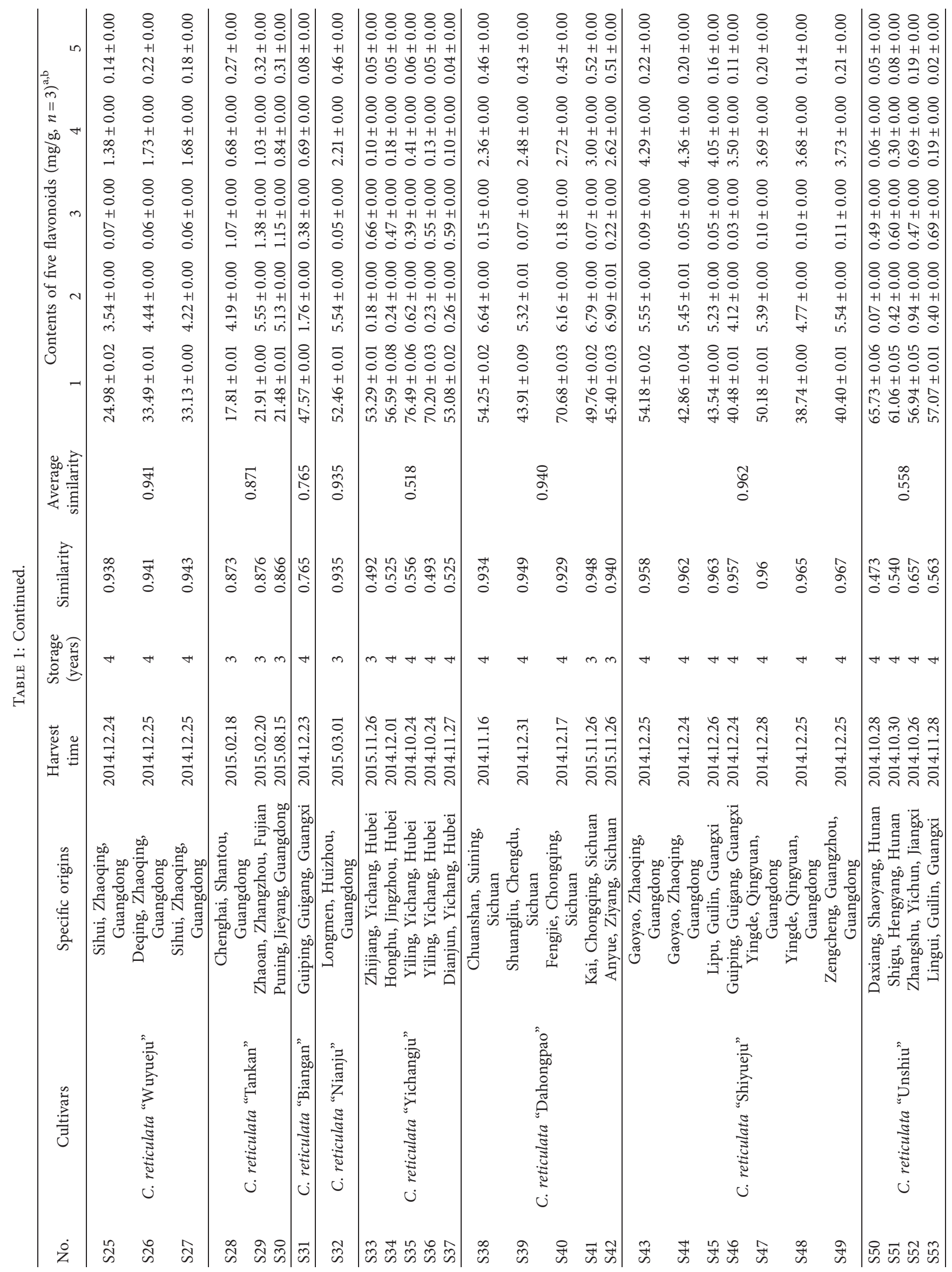




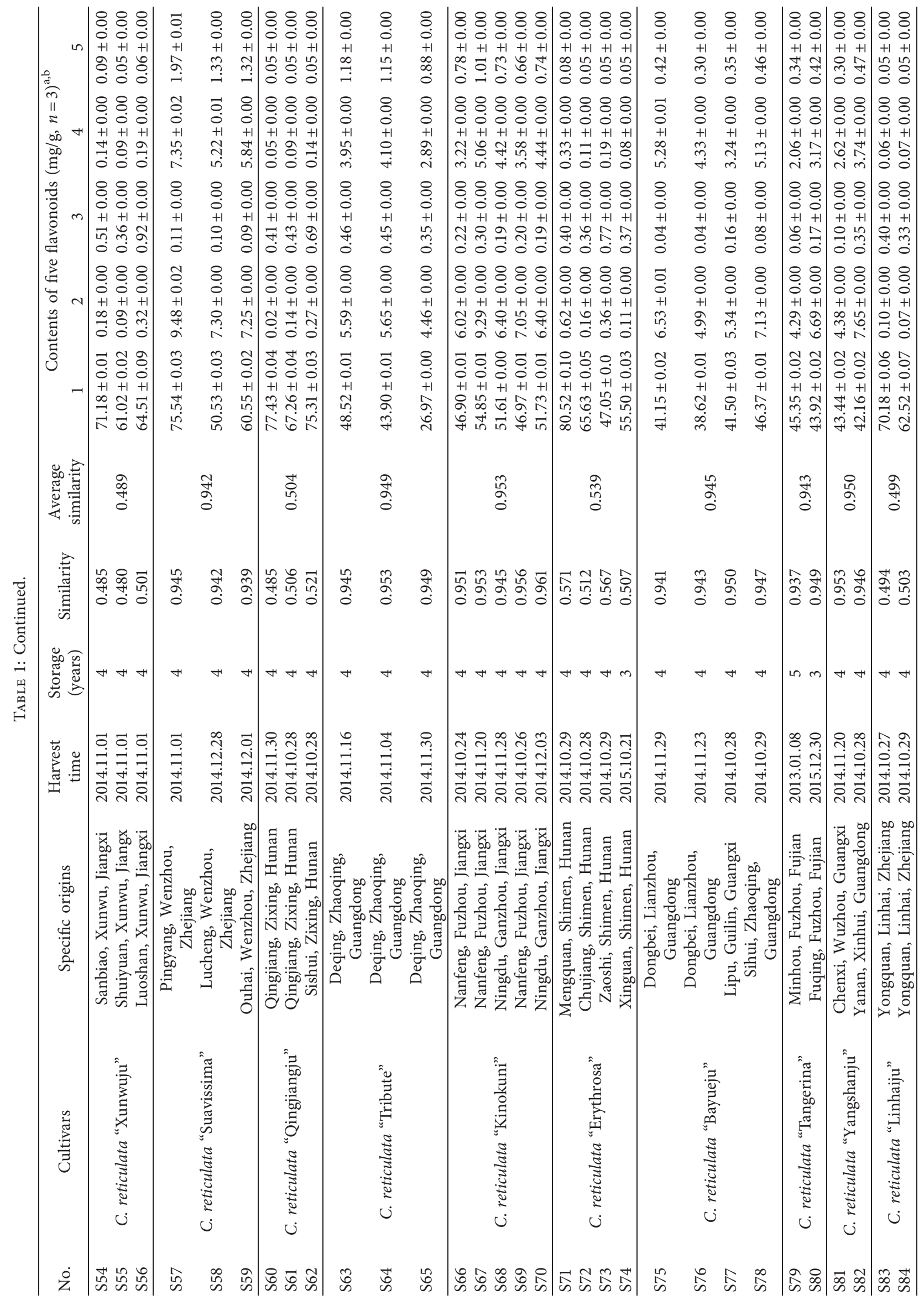




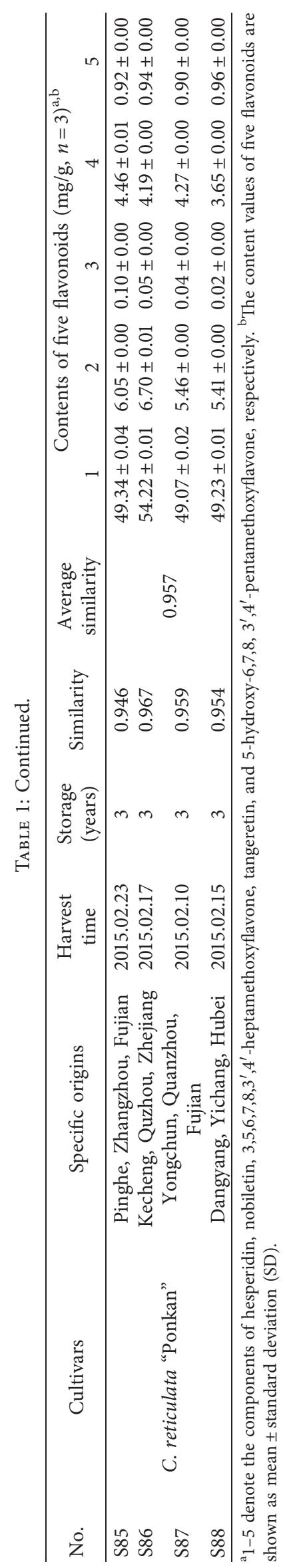


After final administration for an hour, each animal was put in a sealed glass container and exposed to $25 \%$ ammonia water aerosol for $20 \mathrm{~s}$ by an ultrasonic nebulizer [18]. The mice were then removed to observe the incubation period of cough and bouts of cough within 3 minutes.

2.4.2. Expectorant Activity Assay. Kunming mice were divided into five groups ( $n=10 /$ group): control, MECRP-20, MECRP-40, MECRP-80, and AH-40, and each of the groups was medicated orally continuously for 7 days ( 1 time/day). The control group and treated groups were administrated based on the method of the antitussive activity assay, whereas the standard group received $\mathrm{AH} 40 \mathrm{mg} / \mathrm{kg}$ orally. Thirty minutes after the final administration, each mouse was injected intraperitoneally with $0.5 \mathrm{~mL}$ phenol red solution in saline and killed by cervical dislocation 30 minutes later. The tracheas of the same length were taken from the thyroid cartilage to the trachea branch of the mice and washed ultrasonically with $3 \mathrm{~mL} \mathrm{NaOH}$ solution $(0.03 \mathrm{~mol} / \mathrm{L})$ in saline for 10 minutes [19]. Moreover, the absorbance of phenol red in the solution was measured spectrophotometrically at a wavelength of $546 \mathrm{~nm}$, and its content was calculated under the linear regression equation $y=0.1428 x+0.0062\left(R^{2}=0.9993\right)$ (" $\mathrm{x}$ " denotes the concentration of phenol red solution and " $y$ " denotes the absorbance).

2.4.3. Statistical Analysis. The results of activity assays were statistically analyzed by SPSS (Version 16.0) and GraphPad Prism software (Version 5.0), which are expressed as mean \pm standard deviation (SD). One-way ANOVA was used for the data analysis, followed by the LSD test to compare the differences between control and test groups. $P<0.05$ was considered statistically significant in this study.

2.5. HPLC Fingerprint Analysis. The HPLC condition of the MECRP was performed in a previous method described by Luo et al. [17]. SIL-20A HPLC (Shimadzu, Japan) equipped with an SPD-20A UV detector and a Diamonsil C18 column $(250 \times 4.6 \mathrm{~mm}$, i.d. $5 \mu \mathrm{m})$ was used for HPLC analysis. The mobile phase was solution A (water) and solution B (acetonitrile) with the following gradient program: $0-15 \mathrm{~min}$, $15-40 \% \mathrm{~B} ; 15-35 \mathrm{~min}, 40-50 \% \mathrm{~B}$; 35-40 min, 50-75\% B; and 40-50 $\mathrm{min}, 75-85 \% \mathrm{~B}$. Other HPLC conditions are shown as follows: flow rate: $1.0 \mathrm{~mL} / \mathrm{min}$; column temperature: $25^{\circ} \mathrm{C}$; injection volume: $20 \mu \mathrm{L}$; and detection wavelength: $283 \mathrm{~nm}$ and $330 \mathrm{~nm}$. The chromatograms $(330 \mathrm{~nm})$ of C. reticulata "Chachi” samples produced in Xinhui (S1-S12) were firstly imported into the software "Similarity Evaluation System for Chromatographic Fingerprint of Traditional Chinese Medicine (Version 2012)" to establish the GCP fingerprint. With the median method and choosing three chromatographic peaks of hesperidin, nobiletin, and tangeretin for multipoint calibration, a GCP common pattern was generated. Next, the final fingerprint was established according to the representative chromatograms of remaining cultivars (S13-S88) together with the GCP common pattern. The similarity values of different cultivars were finally calculated based on the GCP common pattern.

2.6. LC/MS-IT-TOF Analysis. LC/MS-IT-TOF analysis was carried out as previously reported with a minor modification [20]. The HPLC instrument equipped with the ion-trap and time-of-flight mass spectrometer (Shimadzu, Japan) through electrospray ionization (ESI) was used. The compounds of extraction were isolated from the HPLC system with the same condition as in HPLC analysis mentioned above. The electrospray ionization (ESI) source was operated in a positive ionization mode, and its conditions are listed as follows: nitrogen gas flow, $1.5 \mathrm{~L} / \mathrm{min}$; dry gas temperature, $200^{\circ} \mathrm{C}$; fragmentation voltage, $1.62 \mathrm{kV}$; nebulizer pressure, $45 \mathrm{psi}$; and full and dependent scan data acquisition, $\mathrm{m} / \mathrm{z}$ 100-1000. Ion spectra were recorded from 5 to $40 \mathrm{eV}$ with argon. Data processing was performed using LC/MS Solution 3.60.361 software (Shimadzu), while molecular formula prediction and elemental composition prediction were done using Formula Predictor and Accurate Mass Calculator software, respectively.

2.7. Quantitative Determination and PCA. The components of hesperidin and other four PMFs were detected at $283 \mathrm{~nm}$ and $330 \mathrm{~nm}$ in HPLC, respectively, which were calculated using the external standard method and expressed as mean \pm SD. The contents of five flavonoids in all samples were first normalized by SPSS software (Version 16.0), and then principal component analysis was performed.

\section{Results and Discussion}

3.1. Antitussive and Expectorant Activities of MECRP. The effects of the MECRP on ammonia water-irritated cough (Figures 1(a) and 1(b)) and tracheal phenol red excretion in mice are depicted in Figure 1(c). Statistical analysis showed significant differences between the control group and treated groups in the incubation period of cough $(P<0.05)$, bouts of cough within 3 minutes $(P<0.05)$, and tracheal phenol red excretion $(P<0.05)$. The test of ammonia water-irritated cough showed that the MECRP significantly prolonged the incubation period of cough and decreased the bouts of coughs in mice, possessing a good ability to relieve cough. In addition, the tracheal phenol red excretion test illustrated that the MECRP could effectively reduce sputum with the increase of the excretion of phenol red in the trachea. It has been well suggested that the MECRP exhibited excellent antitussive and expectorant activities in mice.

3.2. Similarity Analysis on "Activity Fingerprint". The GCP fingerprint (Figure 2(a)) was firstly established by twelve batches of traditional genuine herbs from Xinhui (S1-S12) due to their high quality. And a GCP common pattern (Figure 2(b)) was further generated through the characteristics of samples S1-S12. There were 18 common peaks determined in the GCP common pattern, of which peaks 3, 


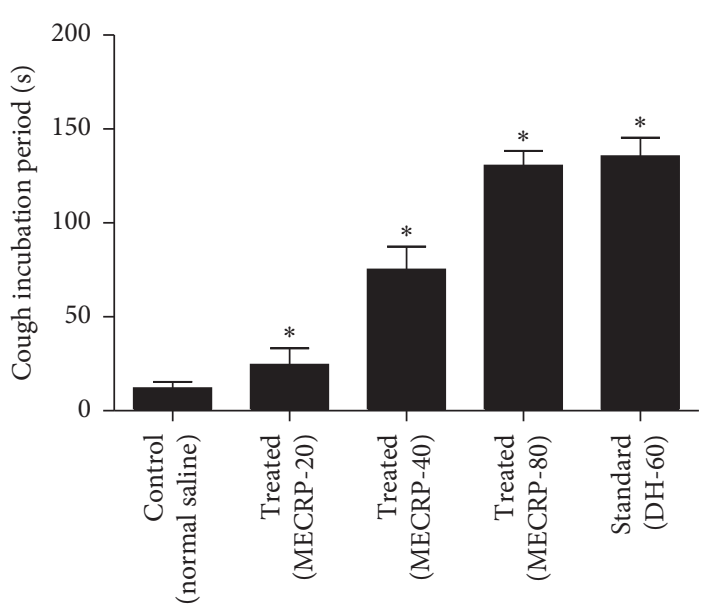

(a)

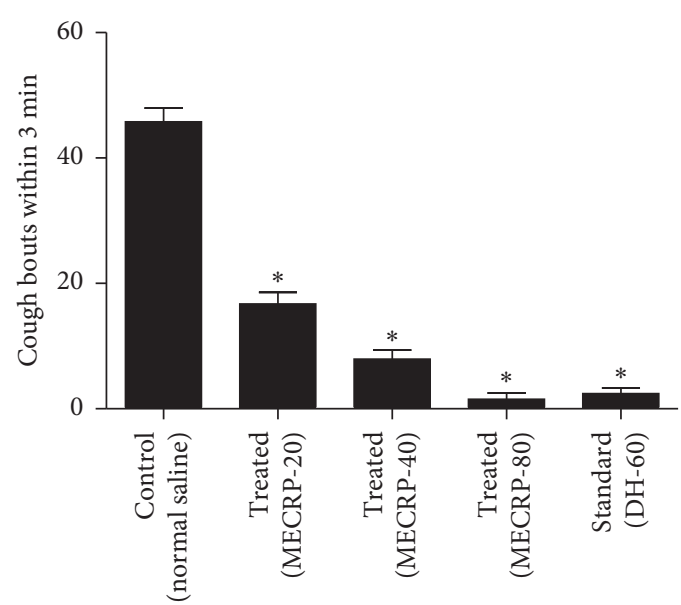

(b)

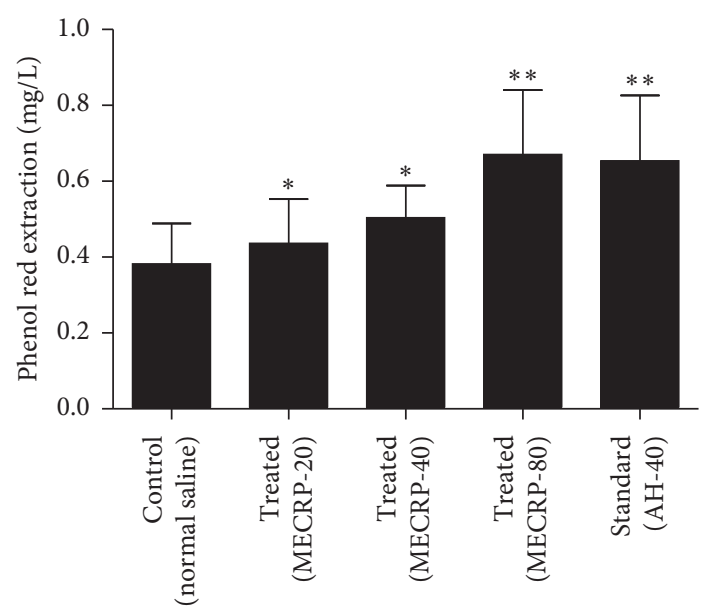

(c)

FIGURE 1: Effect of the MECRP on ammonia water-irritated cough and tracheal phenol red excretion in mice. (a) Incubation period of cough. (b) Bouts of cough. (c) Tracheal phenol red excretion. All values are expressed as mean \pm SD $(n=10) .{ }^{*} P<0.05$ and ${ }^{* *} P<0.01$ compared to the control group (one-way ANOVA followed by the LSD test).

9, and 12 were identified as hesperidin, nobiletin, and tangeretin, respectively. According to statistical analysis, the common peak area of each sample (S1-S12) accounted for $97.276 \%-98.870 \%$ (>90\%) of total peak areas. And all of the accuracy, repeatability, and stability results showed that the RSD of relative retention time and relative peak area of the 18 common peaks was below $3 \%$, satisfying the demands of fingerprint.

The fingerprint of representative cultivars of CRP was finally established with the GCP common pattern (Figure 2(c)). In addition, the similarity evaluation results of 88 samples are shown in Table 1. The similarity values of samples S1-S12 compared to the GCP common pattern were all above 0.990, indicating that the C. reticulata "Chachi" samples from Xinhui were all of good quality uniformly. Moreover, the average similarity of the C. reticulata "Chachi" samples from other areas (S13-S22) was high (0.974), suggesting that there was little characteristic difference among C. reticulata "Chachi" samples. It was also seen that several cultivars of CRP showed high similarity to C. reticulata "Chachi," such as $C$. reticulata "Shiyueju," C. reticulata "Ponkan," C. reticulata "Kinokuni," C. reticulata "Yangshanju," C. reticulata "Tribute," C. reticulata "Bayueju," C. reticulata "Suavissima," C. reticulata "Wuyueju," and C. reticulata "Nianju," with the high average values of $0.962,0.957,0.953,0.950,0.949,0.945,0.942$, 0.941 , and 0.935 , respectively. However, the cultivars of $C$. reticulata "Unshiu," C. reticulata "Erythrosa," C. reticulata "Yichangju," C. reticulata "Qingjiangju," C. reticulata "Linhaiju," and C. reticulata "Xunwuju" showed little similarity to C. reticulata "Chachi," with the low average similarity of 0.558 , $0.539,0.518,0.504,0.499$, and 0.489 , respectively.

3.3. Chemical Profiling on "Activity Fingerprint" by LC/MSIT-TOF. The technology of LC/MS-IT-TOF has been proved to be an effective method for identifying the unknown compounds in materials [21]. The total ion chromatogram (TIC) of CRP sample S1 (a) and mixed standard solution (b) in the positive mode is displayed in Figure 3. After chemical profiling, a total of 17 characteristic 


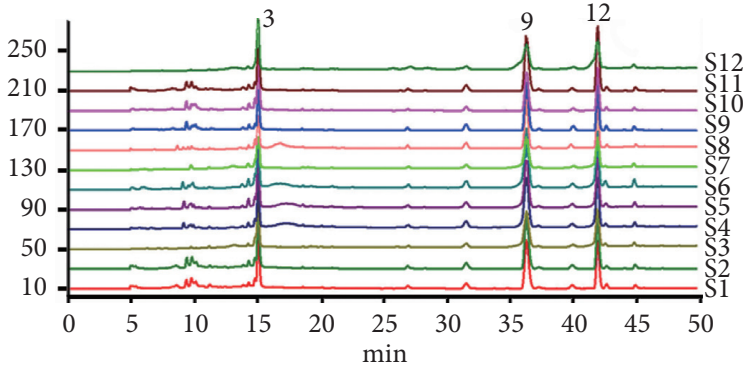

(a)

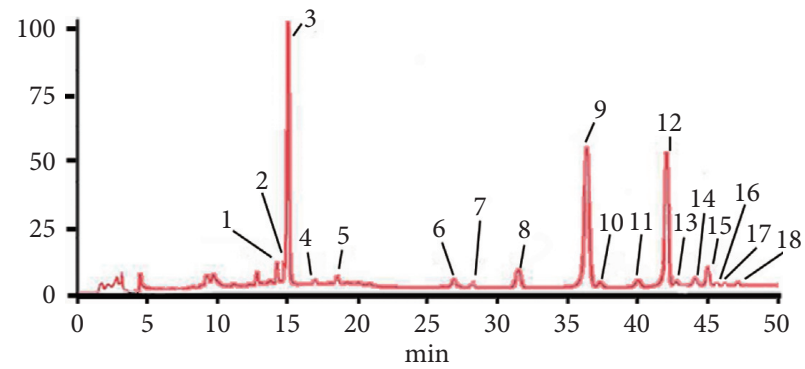

(b)

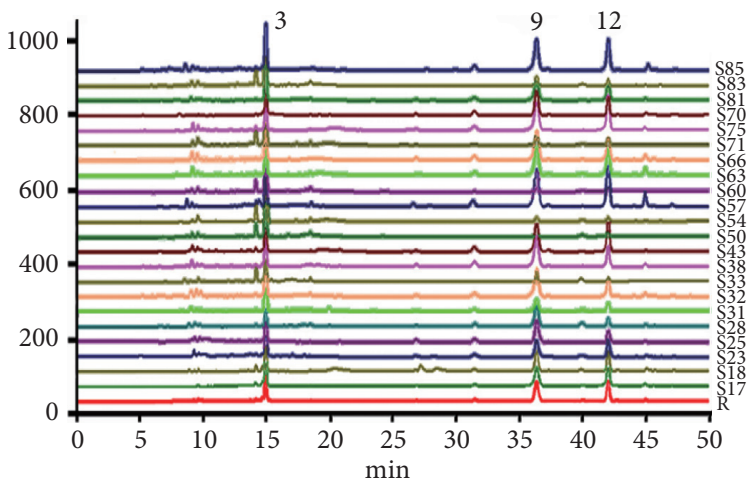

(c)

FIGURE 2: (a) GCP fingerprint of samples S1-S12. (b) GCP common pattern R. (c) Fingerprint of representative cultivars of CRP. Peaks 3, 9, and 12 are hesperidin, nobiletin, and tangeretin, respectively.

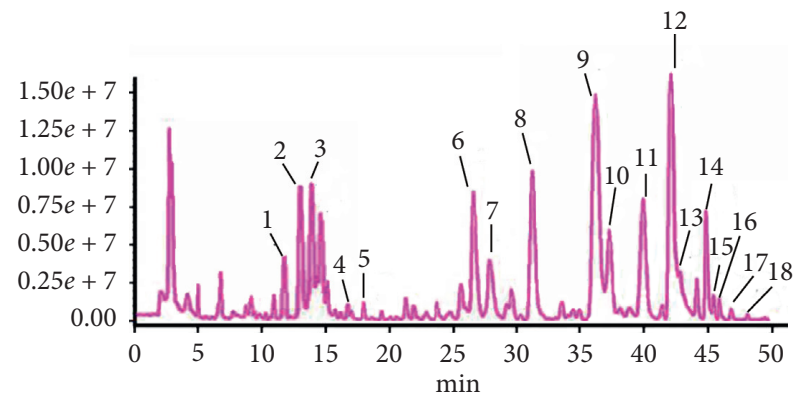

(a)

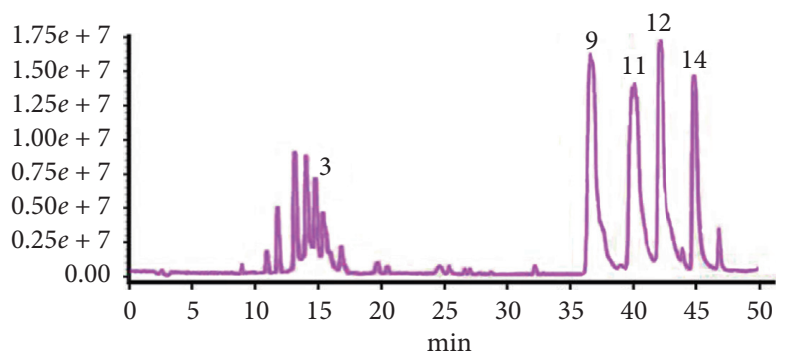

(b)

FIgURE 3: (a) Total ion chromatogram (TIC) of CRP in the positive mode. (b) TIC of mixed standard solution in the positive mode. Peaks 3 , $9,11,12$, and 14 are hesperidin, nobiletin, 3,5,6,7,8,3', $4^{\prime}$-heptamethoxyflavone, tangeretin, and 5-hydroxy-6,7,8,3', $4^{\prime}$-pentamethoxyflavone, respectively.

compounds, including 3 flavonoid glycosides and 14 PMFs, were identified according to the information of mass fragment ions and retention times, and the detailed information is illustrated in Table 2.

3.3.1. Identification of Flavonoid Glycosides. Flavonoids are a kind of organic compounds with C6-C3-C6 as the parent nucleus. Among them, flavonoid glycosides are widely present in citrus, including flavone-O-glycosides and flavone-C-glycosides. The flavone-O-glycosides are generally characterized by attaching glycosidic substituents to the flavonoid skeleton via the C-7 hydroxyl group, while the
flavone-C-glycosides directly linked glycosidic substituents to the $\mathrm{C}$ atoms on the flavone $\mathrm{A}$ ring [22].

Compound 2 displayed a quasimolecular ion at $m / z 581$, which formed a fragment ion at $m / z 273$ due to the loss of terminal Rha-Glc (308 Da). The MS spectrum also showed secondary fragment cracking by aglycon. For instance, the fragment at $m / z 153$ was produced by RDA cleavage, and the fragment at $m / z 245$ was formed corresponding to the flavonoid glycoside ion loss of CO $(28 \mathrm{Da})$. Therefore, the compound was considered naringin according to the mass fragmentation information above [23].

Compound 3 was putatively identified as hesperidin because of the same mass spectrum and chromatographic 


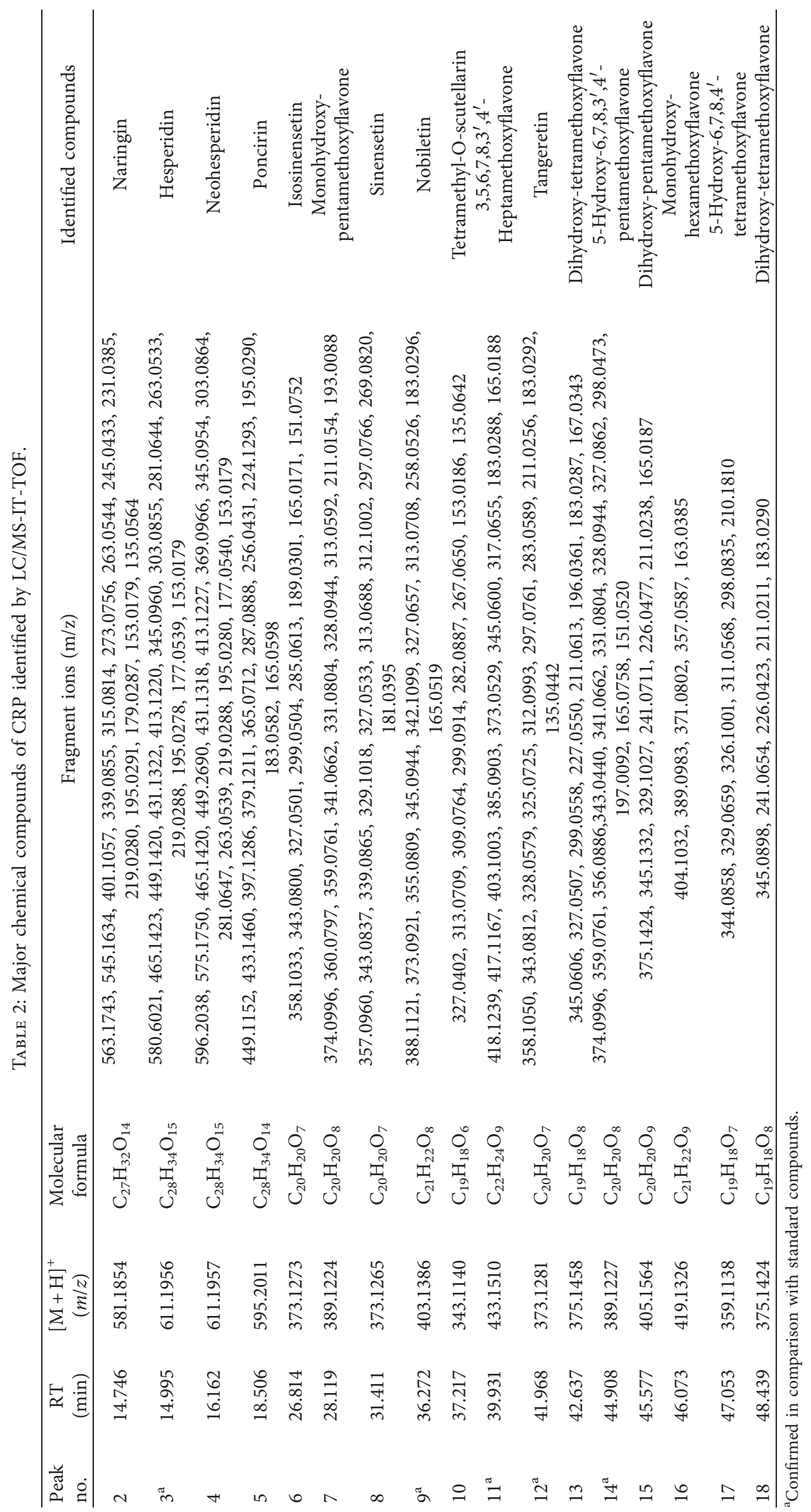


characteristics with the reference standard. The high intensity ions at $\mathrm{m} / z 449$ and 303 were observed on the removal of glucose $(162 \mathrm{Da})$ and rhamnosylglucose $(308 \mathrm{Da})$ from the precursor ion, respectively. An extra minor fragment at $\mathrm{m} / \mathrm{z} 153$ was yielded by the RDA cleavage of aglycone ion. Compound 4 showed the similar fragment information to compound 3 but was different in the relative abundances, which could be inferred as neohesperidin [24].

Compound 5 showed the $[\mathrm{M}+\mathrm{H}]^{+}$ion at $m / z 595$ in the first-order MS spectrum, suggesting the molecular formula of $\mathrm{C}_{28} \mathrm{H}_{34} \mathrm{O}_{14}$. A high-intensity ion at $m / z 287$ after losing neohesperidose $(308 \mathrm{Da})$ was generated. This compound was tentatively inferred as poncirin after the comparison of its mass spectrum data [25].

3.3.2. Identification of PMFs. PMFs are significant flavonoids particularly presented in citrus species, containing multiple methoxyl groups, low polarity, and planar structure [26]. With a basic unit of $222 \mathrm{Da}$, the molecular weights of PMFs vary depending on the amount of methoxyl and hydroxyl groups [27, 28].

Compound 9 was confirmed as nobiletin by comparison to the reference standard with the same fragmentation information. Nobiletin gave an $[\mathrm{M}+\mathrm{H}]^{+}$ion at $\mathrm{m} / z 403$ and yielded fragment ions at $\mathrm{m} / z 388\left(\left[\mathrm{M}+\mathrm{H}-\mathrm{CH}_{3}\right]^{+}\right), 373$ $\left(\left[\mathrm{M}+\mathrm{H}-2 \mathrm{CH}_{3}\right]^{+}\right)$, and $355\left(\left[\mathrm{M}+\mathrm{H}-2 \mathrm{CH}_{3}-\mathrm{H}_{2} \mathrm{O}\right]^{+}\right)$. Similarly, compound 11 was deduced as $3,5,6,7,8,3^{\prime}, 4^{\prime}$ heptamethoxyflavone by comparing to the reference standard with the $[\mathrm{M}+\mathrm{H}]^{+}$ion at $m / z 433$.

Compounds $6,7,8,12,14$, and 15 were considered pentamethoxyflavones based on their molecular formula and fragment ions [29]. Comparing with a reference standard allowed us to identify compound 14 as 5-hydroxy$6,7,8,3,3^{\prime}, 4^{\prime}$-pentamethoxyflavone. It exhibited the parent ion $[\mathrm{M}+\mathrm{H}]^{+}$at $\mathrm{m} / z 389$ and the product fragment ion at $\mathrm{m} / \mathrm{z}$ $374\left(\left[\mathrm{M}+\mathrm{H}-\mathrm{CH}_{3}\right]^{+}\right), 359\left(\left[\mathrm{M}+\mathrm{H}-2 \mathrm{CH}_{3}\right]^{+}\right)$, and 341 $\left(\left[\mathrm{M}+\mathrm{H}-2 \mathrm{CH}_{3}-\mathrm{H}_{2} \mathrm{O}\right]^{+}\right)$. Compounds 6 and 8 were known as isosinensetin and sinensetin combining with the mass data of the previous research [30]. Compound 7 was presumed to be monohydroxy-pentamethoxyflavone based on the molecular formula of $\mathrm{C}_{20} \mathrm{H}_{20} \mathrm{O}_{8}$ and similar fragment of compound 14. Similarly, compound 15 was inferred to be dihydroxy-pentamethoxyflavone differed by the molecular weight of 16 of compound 7 .

Compound 12 was identified as tangeretin by comparing the retention time and fragmentation information of the reference standard. Moreover, the fragmentation information of compound 17 was similar to that of compound 12 and differed by the molecular weight of 14 . Combining with the fragment information previously reported [31], compound 17 was presumed to be 5-hydroxy-6,7,8,4'-tetramethoxyflavone.

3.4. Quality Evaluation by Quantitative Determination and $P C A$. Hesperidin and other four main PMFs selected from 17 characteristic compounds profiled above were determined, and the results are given in Table 1. The method validation showed a good linearity $\left(R^{2}>0.9990\right)$, and the
TABle 3: Component matrix of PCA.

\begin{tabular}{|c|c|c|}
\hline \multirow[t]{2}{*}{ Components } & \multicolumn{2}{|c|}{$\begin{array}{c}\text { Variable } \\
\text { importance in } \\
\text { projection (VIP) } \\
\text { values }\end{array}$} \\
\hline & PC1 & PC2 \\
\hline Hesperidin & -0.255 & 0.953 \\
\hline Nobiletin & 0.934 & -0.121 \\
\hline $3,5,6,7,8,3^{\prime}, 4^{\prime}$-Heptamethoxyflavone & -0.641 & -0.247 \\
\hline Tangeretin & 0.965 & 0.011 \\
\hline 5-Hydroxy-6,7,8, 3', $4^{\prime}$-pentamethoxyflavone & 0.827 & 0.227 \\
\hline
\end{tabular}

2 components were extracted.

RSDs of accuracy, repeatability, stability, and recovery were all below 3\%, indicating that the assay method was feasible.

PCA is a well-known analysis method with the purpose of dimensionality reduction to transform multiple indicators into a few comprehensive indicators $[32,33]$. Defining the contents of the five flavonoids as five characteristics, PCA was used to determine the correlation among samples from different cultivars in this research. The first two principal components were extracted by PCA (Table 3), accounting for $79.979 \%$ of the total variance $(\mathrm{PC} 1=59.269 \%$ and $\mathrm{PC} 2=20.710 \%)$. And the variable importance in projection (VIP) values of nobiletin, tangeretin, 3,5,6,7,8,3', $4^{\prime}$-heptamethoxyflavone, and 5-hydroxy- $6,7,8,3^{\prime}, 4^{\prime}$-pentamethoxyflavone were $0.934,0.965$, -0.641 , and 0.827 , respectively, showing more contribution to PC1, while hesperidin contributed more to PC2 with the VIP value of 0.953 . It was indicated that these five compounds were the characteristic chemicals distinguishing various cultivars of CRP; especially, the four PMFs contributed more to PC1, accounting for $59.269 \%$ of the total variance.

As the result of the PCA plot shows (Figure 4), 88 samples with 21cultivars were categorized into four groups. Group 1 contained C. reticulata "Unshiu," C. reticulata "Erythrosa," C. reticulata "Yichangju," C. reticulata "Qingjiangju," C. reticulata "Linhaiju," C. reticulata "Xunwuju," and C. reticulata "Biangan," with the high content of hesperidin and low PMFs. $C$. reticulata "Tankan" was in group 2 due to its lower content of flavonoids, while C. reticulata "Suavissima" with a higher content of flavonoids was in group 3. Other eleven cultivars were clustered in group 4 with C. reticulata "Chachi," showing parts of similarities and relationships with the traditional genuine herb. The result of PCA was approximately consistent with that of similarity analysis.

Since an LC/MS-IT-TOF was used, 17 characteristic compounds were finally profiled on the 18 common peaks at the "activity fingerprint," and all of them were identified belonging to flavonoids, suggesting that there may be a potential relationship between the differences among varieties and flavonoid compositions. Then, hesperidin and four main PMFs were selected for quantitative determination and further analysis. And the PCA results confirmed that the differences among varieties were significantly correlated with flavonoids, not only including hesperidin, the single indicator for CRP quality control in Chinese Pharmacopoeia (version 2015) [1], but also including four main PMFs, such as

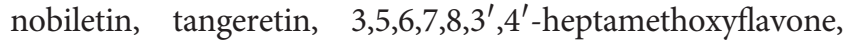




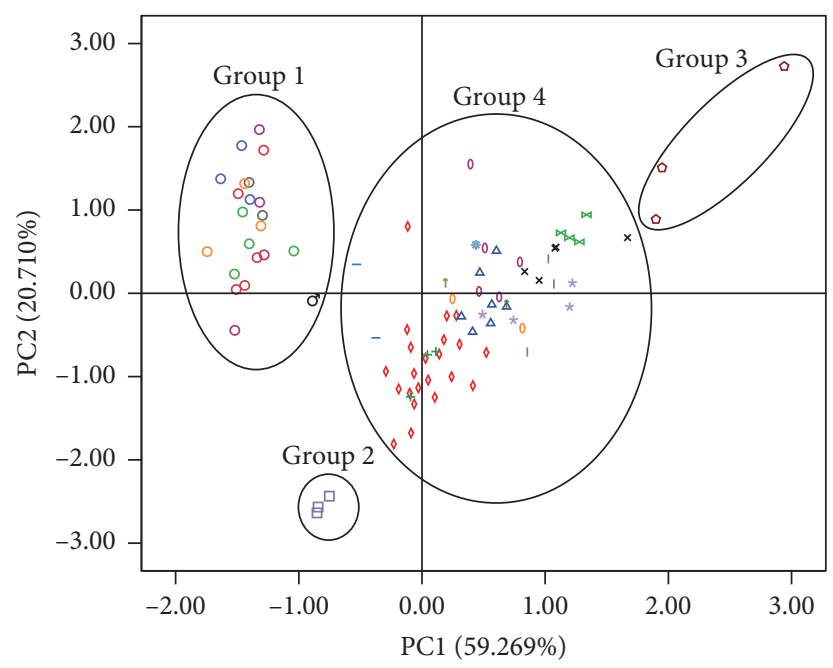

Cultivars

- C. reticulata "Chachi" - C. reticulata "Subcompressa"

† C. reticulata "Tangerina" - C. reticulata "Suavissima"

- C. reticulata "Dahongpao" 口 C. reticulata "Tankan"

+ C. reticulata "Wuyueju" ơ C. reticulata "Biangan"

* C. reticulata "Bayueju" ○ C. reticulata "Erythrosa"

$\Delta$ C. reticulata "Shiyueju" ○ C. reticulata "Linhaiju"

- C. reticulata "Yangshanju" ○ C. reticulata "Qingjiangju"

, C. reticulata "Tribute" ○ C. reticulata "Unshiu"

× C. reticulata "Kinokuni" ○ C. reticulata "Xunwuju"

*C. reticulata "Nianju" ○ C. reticulata "Yichangju"

$\bowtie$ C. reticulata "Ponkan"

FIGURE 4: PCA results of CRP from different cultivars.

and 5-hydroxy-6,7,8,3', $4^{\prime}$-pentamethoxyflavone. Therefore, we could not deny the importance of PMFs in CRP, and it is considerable to add PMFs as indicator components for CRP quality control and identification in Chinese Pharmacopoeia.

\section{Conclusion}

In the present study, an effective "activity fingerprint" on CRP from different cultivars involving antitussive and expectorant activity assays, similarity analysis, LC/MS-ITTOF analysis, quantitative analysis, and PCA was perfectly established, which was proven to relate to pharmacological activity and conducted to find the characteristic chemicals distinguishing various cultivars of CRP for quality control. From the results above, the recorded cultivars of $C$. reticulata "Chachi," C. reticulata "Tangerina," and C. reticulata "Dahongpao" were quite similar to each other except for $C$. reticulata "Unshiu," which indicated that the selection of the traditional medicinal herb of CRP was rational related to the high content of PMFs. In addition, there are some other cultivars significantly similar to C. reticulata "Chachi," such as C. reticulata "Kinokuni," C. reticulata "Yangshanju," C. reticulata "Wuyueju," and C. reticulata "Nianju," and so on. Among them, it was worth noting that the cultivars of $C$. reticulata "Shiyueju," C. reticulata "Ponkan," C. reticulata "Tribute," and C. reticulata "Bayueju" were rarely used for traditional medicine but exhibited a relatively high content of hesperidin and PMFs, even exceeding that of C. reticulata "Chachi." Therefore, these four cultivars can be considered effective resources for the application of CRP, which provide a reference for citrus resource development.

\section{Data Availability}

The data used to support the findings of this study are included within the article.

\section{Conflicts of Interest}

The authors declare that they have no conflicts of interest.

\section{Acknowledgments}

This work was greatly supported by the National Key R\&D Program of China (Nos. 2017YFC1701103 and 2017YFC1701101), National Modern Agricultural Industrial Park of China (No. njf [2017] 110), National Natural Science Foundation of China (No. 31401613), and Cultivation Plan for High-Level University Academic Backbone of Guangzhou Medical University in 2017 (No. gydf [2017] 210).

\section{Supplementary Materials}

Table of abbreviations and acronyms. (Supplementary Materials)

\section{References}

[1] Committee of National Pharmacopoeia, The Pharmacopoeia of the People's Republic of China, Vol. 1, China Medical Science and Technology Press, Beijing, China, 2015.

[2] X. Yu, S. Sun, Y. Guo et al., "Citri Reticulatae Pericarpium (Chenpi): botany, ethnopharmacology, phytochemistry, and pharmacology of a frequently used traditional Chinese medicine," Journal of Ethnopharmacology, vol. 220, pp. 265282, 2018.

[3] W. Zheng, D. Xu, M. Wang, Y. Zhang, Q. Gao, and Y. Gao, "Fingerprint analysis and multi-component determination of ribonucleic acid for injection II recipe by HPLC-DAD and LC-ESI-MS methods," Journal of Chromatographic Science, vol. 57, no. 3, pp. 238-242, 2019.

[4] P.-L. Ding, C.-M. He, Z.-H. Cheng, and D.-F. Chen, "Flavonoids rather than alkaloids as the diagnostic constituents to distinguish Sophorae Flavescentis Radix from Sophorae Tonkinensis Radix et Rhizoma: an HPLC fingerprint study," Chinese Journal of Natural Medicines, vol. 16, no. 12, pp. 951-960, 2018.

[5] Y. Yang, L. Jiang, G. D. Zheng, L. W. Lin, J. L. Chen, and W. Zhou, "HPLC fingerprint of pericarpium citri reticultae from Guangdong province," Zhong Yao Cai, vol. 34, no. 2, pp. 191-195, 2011.

[6] L. Zhu, L. Fang, Z. Li, X. Xie, and L. Zhang, "A HPLC fingerprint study on Chaenomelis Fructus," BMC Chemistry, vol. 13, no. 1, p. 7, 2019.

[7] H. Parhiz, A. Roohbakhsh, F. Soltani, R. Rezaee, and M. Iranshahi, "Antioxidant and anti-inflammatory properties of the citrus flavonoids hesperidin and hesperetin: an updated review of their molecular mechanisms and experimental models," Phytotherapy Research, vol. 29, no. 3, pp. 323-331, 2015.

[8] M. Zhao, C. Li, F. Shen, M. Wang, N. Jia, and C. Wang, "Naringenin ameliorates LPS-induced acute lung injury 
through its anti-oxidative and anti-inflammatory activity and by inhibition of the PI3K/AKT pathway," Experimental and Therapeutic Medicine, vol. 14, no. 3, pp. 2228-2234, 2017.

[9] G. Y. Du, S. W. He, L. Zhang, C. X. Sun, L. D. Mi, and Z. G. Sun, "Hesperidin exhibits in vitro and in vivo antitumor effects in human osteosarcoma MG-63 cells and xenograft mice models via inhibition of cell migration and invasion, cell cycle arrest and induction of mitochondrial-mediated apoptosis," Oncology Letters, vol. 16, no. 5, pp. 6299-6306, 2018.

[10] S. Uesato, H. Yamashita, R. Maeda et al., "Synergistic antitumor effect of a combination of paclitaxel and carboplatin with nobiletin from Citrus depressa on non-small-cell lung cancer cell lines," Planta Medica, vol. 80, no. 6, pp. 452-457, 2014.

[11] R. M. Uckoo, G. K. Jayaprakasha, A. Vikram, and B. S. Patil, "Polymethoxyflavones isolated from the peel of Miaray Mandarin (Citrus miaray) have biofilm inhibitory activity in Vibrio harveyi," Journal of Agricultural and Food Chemistry, vol. 63, no. 32, pp. 7180-7189, 2015.

[12] L.-H. Wang, M.-S. Wang, X.-A. Zeng, X.-M. Xu, and C. S. Brennan, "Membrane and genomic DNA dual-targeting of citrus flavonoid naringenin against Staphylococcus aureus," Integrative Biology, vol. 9, no. 10, pp. 820-829, 2017.

[13] H. Javed, K. Vaibhav, M. E. Ahmed et al., "Effect of hesperidin on neurobehavioral, neuroinflammation, oxidative stress and lipid alteration in intracerebroventricular streptozotocin induced cognitive impairment in mice," Journal of the Neurological Sciences, vol. 348, no. 1-2, pp. 51-59, 2015.

[14] J. Bi, H. Zhang, J. Lu, and W. Lei, "Nobiletin ameliorates isoflurane-induced cognitive impairment via antioxidant, antiinflammatory and anti-apoptotic effects in aging rats," Molecular Medicine Reports, vol. 14, no. 6, pp. 5408-5414, 2016.

[15] Y. O. Agrawal, P. K. Sharma, B. Shrivastava et al., "Hesperidin produces cardioprotective activity via PPAR- $\gamma$ pathway in ischemic heart disease model in diabetic rats," PLoS One, vol. 9, no. 11, Article ID e111212, 2014.

[16] A. M. Mahmoud, R. J. Hernández Bautista, M. A. Sandhu, M. A. Sandhu, and O. E. Hussein, "Beneficial effects of citrus flavonoids on cardiovascular and metabolic health," Oxidative Medicine and Cellular Longevity, vol. 2019, Article ID 5484138, 19 pages, 2019.

[17] M. Luo, H. Luo, P. Hu, Y. Yang, B. Wu, and G. Zheng, "Evaluation of chemical components in Citri Reticulatae Pericarpium of different cultivars collected from different regions by GC-MS and HPLC," Food Science \& Nutrition, vol. 6, no. 2, pp. 400-416, 2018.

[18] T. Guo, J. Qing Wei, and J. Ping Ma, "Antitussive and expectorant activities of Potentilla anserina," Pharmaceutical Biology, vol. 54, no. 5, pp. 807-811, 2016.

[19] K. J. Song, Y.-J. Shin, K. R. Lee, E. J. Lee, Y. S. Suh, and K.-S. Kim, "Expectorant and antitussive effect of Hedera helixand rhizoma coptidis extracts mixture," Yonsei Medical Journal, vol. 56, no. 3, pp. 819-824, 2015.

[20] G.-D. Zheng, P. Zhou, H. Yang, Y.-S. Li, P. Li, and E.-H. Liu, "Rapid resolution liquid chromatography-electrospray ionisation tandem mass spectrometry method for identification of chemical constituents in Citri Reticulatae Pericarpium," Food Chemistry, vol. 136, no. 2, pp. 604-611, 2013.

[21] Y. Liang, Y.-Y. Zhou, Y.-N. Liu et al., "Study on the plasma protein binding rate of Schisandra lignans based on the LCIT-TOF/MS technique with relative quantitative analysis," Chinese Journal of Natural Medicines, vol. 11, no. 4, pp. 442-448, 2013.

[22] S. S. Li, J. Wu, L. G. Chen et al., "Biogenesis of C-glycosyl flavones and profiling of flavonoid glycosides in lotus
(Nelumbo nucifera)," PLoS One, vol. 9, no. 10, Article ID e108860, 2014.

[23] K. Luo and F. Feng, "Identification of absorbed components and metabolites of Zhi-Zi-Hou-Po decoction in rat plasma after oral administration by an untargeted metabolomicsdriven strategy based on LC-MS," Analytical and Bioanalytical Chemistry, vol. 408, no. 21, pp. 5723-5735, 2016.

[24] Z. Lin, H. Wang, Y. Xu, J. Dong, Y. Hashi, and S. Chen, "Identification of antioxidants in Fructus aurantii and its quality evaluation using a new on-line combination of analytical techniques," Food Chemistry, vol. 134, no. 2, pp. 1181-1191, 2012.

[25] X. Liu, Z. Gu, Y. Guo et al., "Rapid analysis of Aurantii Fructus Immaturus (Zhishi) using paper spray ionization mass spectrometry," Journal of Pharmaceutical and Biomedical Analysis, vol. 137, pp. 204-212, 2017.

[26] D.-Y. Zhou, Q. Xu, X.-Y. Xue, F.-F. Zhang, and X.-M. Liang, "Characterization of polymethoxylated flavones in Fructus aurantii by off-line two-dimensional liquid chromatography/ electrospray ionization-ion trap mass spectrometry," Journal of Pharmaceutical and Biomedical Analysis, vol. 49, no. 2, pp. 207-213, 2009.

[27] J.-Y. Zhang, N. Li, Y.-Y. Che et al., "Characterization of seventy polymethoxylated flavonoids (PMFs) in the leaves of Murraya paniculata by on-line high-performance liquid chromatography coupled to photodiode array detection and electrospray tandem mass spectrometry," Journal of Pharmaceutical and Biomedical Analysis, vol. 56, no. 5, pp. 950961, 2011.

[28] H. G. Kim, G.-S. Kim, S. Park et al., "Flavonoid profiling in three citrus varieties native to the Republic of Korea using liquid chromatography coupled with tandem mass spectrometry: contribution to overall antioxidant activity," Biomedical Chromatography, vol. 26, no. 4, pp. 464-470, 2012.

[29] J.-Y. Zhang, Q. Zhang, H.-X. Zhang, Q. Ma, J.-Q. Lu, and Y.-J. Qiao, "Characterization of polymethoxylated flavonoids (PMFs) in the peels of "Shatangju" Mandarin (Citrus reticulata Blanco) by online high-performance liquid chromatography coupled to photodiode array detection and electrospray tandem mass spectrometry," Journal of Agricultural and Food Chemistry, vol. 60, no. 36, pp. 9023-9034, 2012.

[30] H. Kim, J. Y. Moon, A. Mosaddik, and S. K. Cho, "Induction of apoptosis in human cervical carcinoma HeLa cells by polymethoxylated flavone-rich Citrus grandis Osbeck (Dangyuja) leaf extract," Food and Chemical Toxicology, vol. 48, no. 8-9, pp. 2435-2442, 2010.

[31] H.-F. Chen, W.-G. Zhang, J.-B. Yuan, Y.-G. Li, S.-L. Yang, and W.-L. Yang, "Simultaneous quantification of polymethoxylated flavones and coumarins in Fructus aurantii and Fructus aurantii immaturus using HPLC-ESI-MS/MS," Journal of Pharmaceutical and Biomedical Analysis, vol. 59, pp. 90-95, 2012.

[32] M. Jiang, Y. Jiao, Y. Wang et al., "Quantitative profiling of polar metabolites in herbal medicine injections for multivariate statistical evaluation based on independence principal component analysis," PLoS One, vol. 9, no. 8, Article ID e105412, 2014.

[33] X.-L. Cheng, Q.-Q. Zhang, M.-H. Li et al., "Identification of four cornua by ultra-performance liquid chromatography with time-of-flight mass spectrometry coupled with principal component analysis," Journal of Separation Science, vol. 40, no. 8, pp. 1667-1673, 2017. 